\title{
Networks of silicon nanowires: A large-scale atomistic electronic structure analysis
}

\author{
Ümit Keleş, ${ }^{1}$ Bartosz Liedke,${ }^{2}$ Karl-Heinz Heinig, ${ }^{2}$ and Ceyhun Bulutay ${ }^{1, a)}$ \\ ${ }^{1}$ Department of Physics, Bilkent University, Bilkent, Ankara 06800, Turkey \\ ${ }^{2}$ Helmholtz-Zentrum Dresden - Rossendorf, Bautzner Landstr. 400, 01328 Dresden, Germany
}

(Received 30 August 2013; accepted 26 October 2013; published online 11 November 2013)

\begin{abstract}
Networks of silicon nanowires possess intriguing electronic properties surpassing the predictions based on quantum confinement of individual nanowires. Employing large-scale atomistic pseudopotential computations, as yet unexplored branched nanostructures are investigated in the subsystem level as well as in full assembly. The end product is a simple but versatile expression for the bandgap and band edge alignments of multiply-crossing Si nanowires for various diameters, number of crossings, and wire orientations. Further progress along this line can potentially topple the bottom-up approach for Si nanowire networks to a top-down design by starting with functionality and leading to an enabling structure. (C) 2013 AIP Publishing LLC. [http://dx.doi.org/10.1063/1.4830039]
\end{abstract}

The sway of silicon technology on the industrial-scale fabrication generally fosters developments within the same material paradigm of silicon and its native oxide. In this respect, under the pressing demands on functionality and reconfigurability, silicon-based nano-networks (SiNets) with their added dimensional and architectural degrees of freedom will undoubtedly be embraced by the semiconductor community. ${ }^{1}$ The looming appearance of SiNets hinges upon the advancements made on the synthesis of silicon nanowires (NWs) within the past decade. ${ }^{2-6}$ En route, branched nanocrystals, ${ }^{7}$ branched, ${ }^{8-10}$ and tree-like ${ }^{11}$ NWs were realized, followed by the connection of these individual branched nanostructures into large-scale nanowire networks, ${ }^{12,13}$ in some cases using other semiconductors. In particular to SiNets, recently several synthesis procedures have been accomplished which are employed in the fabrication of thermoelectric devices, ${ }^{14}$ biosensors, ${ }^{15}$ and photodetectors. ${ }^{16}$

Notwithstanding, there appears to be a very limited understanding of how to tune the electronic properties of NW networks, which is further compounded by the electrical contact design requirements for proper band alignments. As the encompassing gist of such technical issues, essentially we need to know how will the promising NW utilities be taken over to networks and will networks reveal even new features? These questions form the aspiration of our work. In no doubt, only after having a solid understanding of the underlying electronic properties, can one suggest optimum SiNet morphologies tailored to specific functionalities.

Experimental difficulties on determining the electronic properties of nanostructures call for realistic computational tools with predictive capabilities. For Si NWs, many electronic structure calculations already exist in the literature. ${ }^{17-25}$ A comprehensive review on the theoretical investigations about Si NWs is given by Rurali. ${ }^{26}$ In contrast to the single $\mathrm{Si}$ NWs, hitherto, only a few theoretical studies have been carried out for the electronic properties of branched Si NWs and virtually none on SiNets. Menon et al. ${ }^{27}$ investigated branched pristine Si NWs whereas actual grown NWs have always passivated surfaces. Avramov et al. ${ }^{28}$ considered some very small

${ }^{a}$ Electronic mail: bulutay@fen.bilkent.edu.tr size flower-like Si nanocrystals rather than branched Si NWs. The lack of more realistic theoretical attempts in such an experimentally attractive area can be explained by the fact that even the smallest branched systems, including surface passivation, contains $\sim 10^{3}$ to $10^{4}$ atoms in the computational supercell. The comprehensive study of such structures is one of the prevailing challenges for the first-principles methods due to drastically increased computational load which inevitably invites more feasible semiempirical techniques.

The aim of this study is to lay the groundwork in the level of single-particle semiempirical atomistic pseudopotentials ${ }^{29}$ for SiNets embedded in $\mathrm{SiO}_{2}$ within a restricted energy range around the bandgap. For this purpose, first, we consider Si NWs oriented in $\langle 100\rangle,\langle 110\rangle,\langle 111\rangle$, and $\langle 112\rangle$ crystalline directions. We calculate their energy gaps as well as valence and conduction band edge alignments with respect to bulk $\mathrm{Si}$ as a function of wire diameter. This is followed by a detailed analysis of two- and three-dimensional SiNets to establish an understanding of their electronic properties. Comprehensive results are consolidated into a general expression which provides a simple way to estimate the electronic properties of SiNets.

In our computational framework, the semiempirical pseudopotential-based atomistic Hamiltonian is solved using an expansion basis formed by the linear combination of bulk bands (LCBB) of the constituents of the nanostructure, i.e., $\mathrm{Si}$ and $\mathrm{SiO}_{2}{ }^{30,31}$ While non-self-consistent in nature, semiempirical techniques have the benefit that the calculated bandgaps of nanostructures inherently agree with the experimental values. ${ }^{25,29}$ The surface passivation is provided by embedding the NW structures into an artificial wide bandgap host matrix which is meant to represent silica. ${ }^{32}$ In particular, the embedding matrix has the same band edge line up and dielectric constant as silica, but it is lattice-matched with the diamond structure of $\mathrm{Si}$ (for details, see the supplementary material $^{33}$ ). Although missing surface relaxation and strain effects, the competence of our method has been validated, in the context of embedded Si and Ge nanocrystals, confronting with experimental data for the linear ${ }^{32}$ and third-order nonlinear optical properties ${ }^{34,35}$ and the quantum-confined Stark effect. $^{36}$ 

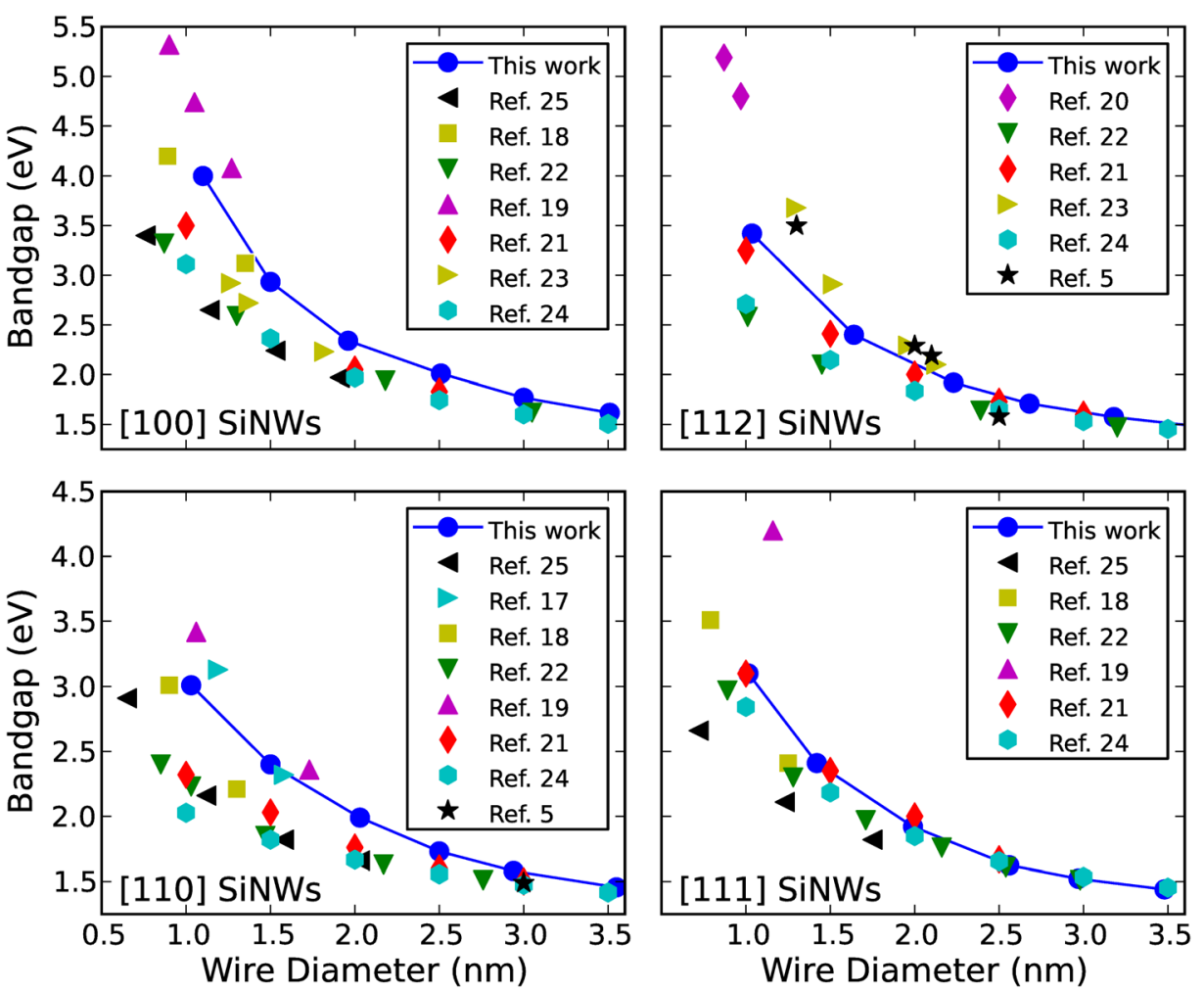

FIG. 1. Bandgap energies as a function of diameter for [100], [112], [110], and [111]-oriented Si NWs. Our results for oxide-passivated NWs (solid lines with filled circles) are compared with data of experimental ${ }^{5}$ and theoretical results of $\mathrm{H}$-passivated NWs including empirical pseudopotential method, ${ }^{25}$ semiempirical tight binding, ${ }^{24}$ and density functional theory calculations correcting bandgaps with GW approximation, ${ }^{17-20}$ hybrid functionals, ${ }^{21,22}$ or scissors operation. $^{23}$
To set the stage for SiNets, first we establish the single NW case. For oxide-passivated Si NWs aligned along the $\langle 100\rangle,\langle 110\rangle,\langle 111\rangle$, and $\langle 112\rangle$ crystalline orientations, we report the effective electronic bandgaps, regardless of whether it is direct or indirect. In Fig. 1, our NW results are compared with a compilation of some representative experimental and theoretical data for wire diameters in the range of $0.5 \mathrm{~nm}-3.5 \mathrm{~nm}$. Note that a strict comparison will not be meaningful as the Si NWs considered here are embedded in $\mathrm{SiO}_{2}$ whereas the literature values are for $\mathrm{H}$-passivated $\mathrm{Si}$ NWs. In general terms, oxide passivation is observed to show similar trends like that of H-passivation.

In Fig. 2, we display our bandgap results for different NW orientations. For all directions, the gaps decrease asymptotically towards the bulk $\mathrm{Si}$ value with increasing

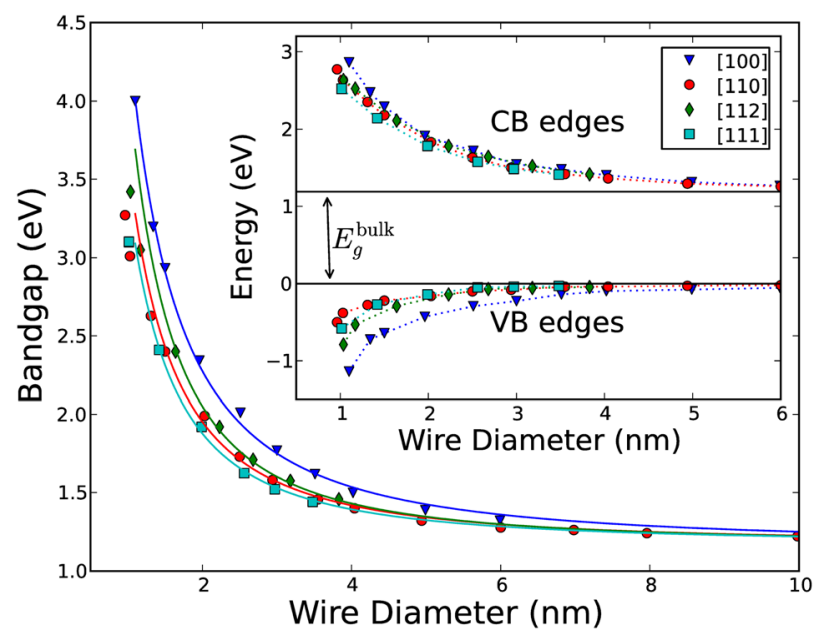

FIG. 2. Bandgap energy of oxide-passivated Si NWs as a function of diameter. The values are fitted with a $C d^{-\alpha}$ form. Inset shows the variation of valence and conduction band edges. The dashed lines of the inset are just guides to the eyes. wire diameter, reflecting the reduction of the quantum confinement effect. In this figure, dependence of the bandgap $E_{g}$ on the wire diameter is described by

$$
E_{g}=E_{g}^{\mathrm{bulk}}+C d^{-\alpha}
$$

as proposed according to the effective mass approximation. ${ }^{37}$ In this expression, $d$ is the diameter of the wire, $C$ and $\alpha$ are fitting parameters, and $E_{g}^{\text {bulk }}=1.17 \mathrm{eV}$ is the experimental bulk Si bandgap value. The fitted $\{C, \alpha\}$ parameters are listed in Table I. In the fitting procedure, we include data points for diameters above $\sim 1.3 \mathrm{~nm}$. This is based on our observation that in the excluded strong confinement regime a different physical mechanism sets in. Namely, the wave function penetrates into the oxide matrix, thereby experiencing a larger effective diameter. ${ }^{23}$ For this reason, in Fig. 2 the data points of smaller diameters somewhat deviate from the fitting curves.

We can note that the hallmark of the quantum confinement within the effective mass approximation is the $1 / d^{2}$ scaling of the single-particle state energies. ${ }^{38}$ The same behavior prevails even when valence band coupling and

TABLE I. Wire orientation-dependent fitting parameters associated with Eq. (1) (also used for Eq. (2)) for the main gap energy $(C, \alpha)$ as well as for VB $\left(C_{\mathrm{VBE}}, \alpha_{\mathrm{VBE}}\right)$ and $\mathrm{CB}\left(C_{\mathrm{CBE}}, \alpha_{\mathrm{CBE}}\right)$ edge energies. When diameters $d$ in Eqs. (1) and (2) are in nm units, the energies come out in units of $\mathrm{eV}$.

\begin{tabular}{lrrrr}
\hline \hline & $\langle 100\rangle$ & $\langle 110\rangle$ & $\langle 111\rangle$ & $\langle 112\rangle$ \\
\hline$C$ & 3.31 & 2.47 & 2.25 & 2.98 \\
$\alpha$ & 1.57 & 1.66 & 1.67 & 1.76 \\
$C_{\mathrm{VBE}}$ & -1.22 & -0.45 & -0.49 & -0.69 \\
$\alpha_{\mathrm{VBE}}$ & 1.64 & 1.66 & 1.94 & 1.98 \\
$C_{\mathrm{CBE}}$ & 2.11 & 2.07 & 1.74 & 2.25 \\
$\alpha_{\mathrm{CBE}}$ & 1.63 & 1.75 & 1.66 & 1.74 \\
\hline \hline
\end{tabular}


conduction band off- $\Gamma$ minima are taken to account in the underlying band structure. ${ }^{39}$ On the other hand, in our analysis the exponent, $\alpha$ significantly deviates from 2 to values in the range 1.57-1.76 depending on the direction (Table I). Discrepancy stems from the lack of atomistic potentials in the former that relies solely on the effective mass and the kinetic energy of the carriers. ${ }^{38}$ Our assertion is that an atomistic treatment becomes crucial even close to the band edge energies.

In the inset of Fig. 2, we plot the variation of valence band (VB) and conduction band (CB) edges as a function of wire diameter for different wire orientations. Indicating the bulk Si band edges with horizontal solid lines, this figure also illustrates the alignments of band edges of Si NWs with respect to bulk Si for increasing diameters. We find out that the band edge energies have the same functional dependence with respect to NW diameter just like the bandgaps, as in Eq. (1). Hence, setting the bulk VB maximum of Si to zero, VB and $\mathrm{CB}$ edge energies can also be described by $E_{\mathrm{VBE}}$ $=C_{\mathrm{VBE}} d^{-\alpha_{\mathrm{VBE}}} \quad$ and $E_{\mathrm{CBE}}=E_{g}^{\text {bulk }}+C_{\mathrm{CBE}} d^{-\alpha_{\mathrm{CBE}}}$, where $\left\{C_{\mathrm{VBE}}, \alpha_{\mathrm{VBE}}\right\}$ and $\left\{C_{\mathrm{CBE}}, \alpha_{\mathrm{CBE}}\right\}$ are fitted for the data points given in the inset of Fig. 2 and listed in Table I. Notably, $\alpha_{\mathrm{CBE}}$ behaves similar to the bandgap exponents $\alpha$, with either one being relatively less sensitive to wire directions. In striking contrast, $\alpha_{\mathrm{VBE}}$ displays a curious dual character: $\langle 111\rangle$ and $\langle 112\rangle$ wires have values around 2 whereas $\langle 100\rangle$ and $\langle 110\rangle$ substantially deviate from the quadratic behavior. ${ }^{40,43}$ Considering device applications, in the supplementary material, ${ }^{33}$ we also provide the band offsets of Si NWs with respect to bulk $\mathrm{Si}$ and $\mathrm{SiO}_{2}{ }^{33}$

Regarding the H-passivated Si NWs, it is known that at a given wire diameter up to around $3 \mathrm{~nm}$, the bandgap follows the ordering $E_{g}^{\langle 100\rangle}>E_{g}^{\langle 111\rangle} \sim E_{g}^{\langle 112\rangle}>E_{g}^{\langle 110\rangle}$ with NW orientation. $^{22,26}$ In our oxide-passivation case, although $\langle 100\rangle \mathrm{Si}$ NWs have the largest bandgap energy as before, the ordering changes to $E_{g}^{\langle 100\rangle}>E_{g}^{\langle 112\rangle}>E_{g}^{\langle 110\rangle} \sim E_{g}^{\langle 111\rangle}$ (see Fig. 2). This ordering is robust under several different pseudopotential parameterizations that we tried for the oxide matrix. Moreover, this observation is in accord with the results of Ref. 41, where the electronic structure of oxide-sheathed $\mathrm{Si}$ NWs is compared with reference H-passivated Si NWs, and it is reported that the magnitude of $E_{g}$ shrinks for $\langle 100\rangle$ and $\langle 111\rangle$-oriented SiNWs while it increases for both $\langle 110\rangle$ and $\langle 112\rangle$-oriented SiNWs. Given the fact that only one calculation was provided in that work for each orientation, more extensive first-principles calculations are required to extract general trends on the ordering of bandgaps of oxidized $\mathrm{Si}$ NWs with wire alignment.

Yan et al. ${ }^{19}$ and Niquet et $a{ }^{24}$ give the VB and CB edges for $\mathrm{H}$-passivated $\mathrm{Si} \mathrm{NWs}$ which agree with the general trends of our VB edge energies (see the inset of Fig. 2). However, for the $\mathrm{CB}$ edge energies of $\langle 112\rangle$ and $\langle 110\rangle \mathrm{Si}$ NWs, they report a smaller variation with diameter (even less than $0.5 \mathrm{meV}$ for $\langle 110\rangle \mathrm{Si} \mathrm{NWs}$ ) while in our case, those values change as much as the $\mathrm{CB}$ edges of $\langle 100\rangle$ and $\langle 111\rangle$ Si NWs. This behavior of $\langle 112\rangle$ and $\langle 110\rangle$ Si NWs gives rise to the distinct bandgap anisotropy of our oxide-passivated $\mathrm{Si}$ NWs.

Next, we consider the crossings of Si NWs as building blocks of SiNets (see Fig. 3). The branched Si NWs
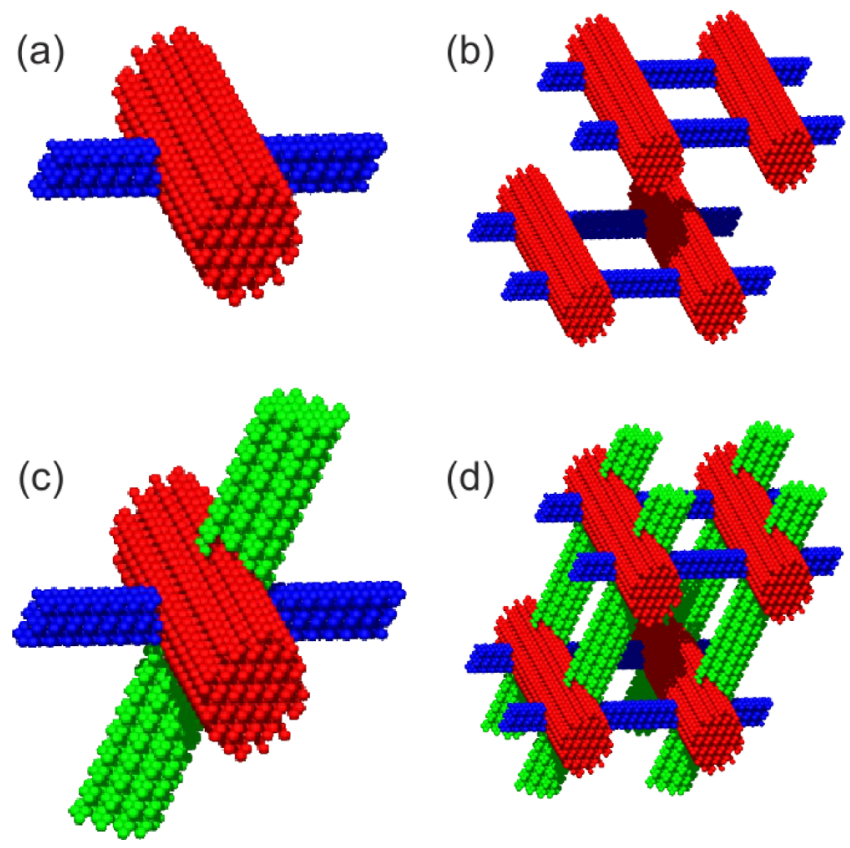

FIG. 3. The computational supercells contain (a) two- or (c) three-wire crossings to build up (b) two- or (d) three-dimensional continuous SiNets, respectively. The wire orientations are in the family of $\langle 110\rangle$ directions. For clarity, Si atoms are shown in different colors for the crossing wires, and the matrix atoms are not shown.

synthesized so far have the tendency to grow in the $\langle 111\rangle$ crystal directions with larger diameters $(>20 \mathrm{~nm}),{ }^{8,9,11}$ However, at sub-10 $\mathrm{nm}$ diameters the occurrence of $\langle 110\rangle$ NW alignment trumps over other directions. ${ }^{4,6,42}$ Henceforth, our results, referring to sub-10 $\mathrm{nm}$ diameter crossings of $\mathrm{Si}$ NWs, are mainly quoted for the crossings of $\langle 110\rangle$-oriented NWs. Nevertheless, we have performed calculations for crossings of $\langle 100\rangle,\langle 110\rangle,\langle 111\rangle$, and $\langle 112\rangle$ aligned NWs as well. Thus, our general conclusions are valid for all directions unless stated otherwise.

As Figs. 3(a) and 3(c) show, our computational supercells contain crossings of two or three NWs. Taking into account the periodic boundary conditions, the calculations are performed for regular arrays of crossings (Figs. 3(b) and $3(d)$ ). These structures are again embedded into the oxide matrix. We note that in comparison to single NW calculations, network supercells require much more atoms. For instance, in the supercell of a crossing of three $3 \mathrm{~nm}$-thick NWs, the supercell consists of $3694 \mathrm{Si}$ and 7970 matrix atoms, respectively. We consider unrelaxed crossings of $\mathrm{Si}$ NWs, i.e., no changes of crossing morphologies by interface energy minimization are taken into account. Although the realistic crossings have some reconstructions, the electron microscopy images show that the intercrossing regions are still very close to the ideal unrelaxed case. ${ }^{8-10}$

The results for various combinations are summarized in Table II. Initially, to unambiguously address the effect of crossing, we check the case when the participating NWs do not cross each other (data shown in brackets in Table II) and are separated by at least $1 \mathrm{~nm}$ to suppress their interactions. We observe that (i) the number of NWs in the supercell does not alter the calculated bandgap when the diameters are equal (Nos. 1-3 and 4-6), (ii) the bandgap is that of the thickest NW when the diameters are distinct. On the other 
TABLE II. Bandgap energies for Si NWs (Nos. 1, 4, 7), two-wire (Nos. 2, 5, 8,9 ), and three-wire crossings (Nos. 3, 6, 10-14). Non-crossing bandgap value for each case is quoted in brackets.

\begin{tabular}{lccccc}
\hline \hline & \multicolumn{3}{c}{ Wire diameters } & & \\
\cline { 2 - 4 } No. & $d_{1}(\mathrm{~nm})$ & $d_{2}(\mathrm{~nm})$ & $d_{3}(\mathrm{~nm})$ & & $E_{g}(\mathrm{eV})$ \\
\hline 1 & 1.50 & $\ldots$ & $\ldots$ & 2.40 & $\ldots$ \\
2 & 1.50 & 1.50 & $\ldots$ & 2.18 & {$[2.40]$} \\
3 & 1.50 & 1.50 & 1.50 & 2.11 & {$[2.40]$} \\
4 & 2.03 & $\ldots$ & $\ldots$ & 1.99 & $\ldots$ \\
5 & 2.03 & 2.03 & $\ldots$ & 1.84 & {$[1.99]$} \\
6 & 2.03 & 2.03 & 2.03 & 1.73 & {$[1.99]$} \\
7 & 2.94 & $\ldots$ & $\ldots$ & 1.57 & $\ldots$ \\
8 & 1.50 & 2.03 & $\ldots$ & 1.96 & {$[1.99]$} \\
9 & 1.50 & 2.94 & $\ldots$ & 1.57 & {$[1.57]$} \\
10 & 1.50 & 1.50 & 2.03 & 1.85 & {$[1.99]$} \\
11 & 1.50 & 2.03 & 2.03 & 1.81 & {$[1.99]$} \\
12 & 1.50 & 1.50 & 2.94 & 1.57 & {$[1.57]$} \\
13 & 1.50 & 2.03 & 2.94 & 1.56 & {$[1.57]$} \\
14 & 2.03 & 2.03 & 2.94 & 1.55 & {$[1.57]$} \\
\hline \hline
\end{tabular}

hand, in the case of crossing NWs, the bandgap values reduce with the increasing number of crossing wires (Nos. 1-3 and 4-6). This is caused by the reduction in confinement due to the wave function extensions into the branches. Here, in going from single NW to two-wire crossing, a significant reduction in the bandgap occurs which becomes not as pronounced when an additional third crossing is introduced (Nos. 1-3). Other aspects of crossing can be discussed referring Nos. 8 and 9: while the bandgap is determined by the thicker NW in the latter, the thinner NW still influences a marginal reduction in the former. Similar behavior is valid for the crossings of three NWs, that is, the bandgap is dominated by the thickest NW while other NWs exert a reduction to the extent of their diameters.

To estimate the bandgap values of SiNets, our observations on NW crossings can be consolidated into a generalized form of Eq. (1). As our main result, we propose for the bandgap $E_{g}$ of $N$ crossing wires, the expression

$$
E_{g}=E_{g}^{\text {bulk }}+C\left(\sum_{i=1}^{N} d_{i}^{\beta}\right)^{-\alpha / \beta}
$$

where $E_{g}^{\text {bulk }}$ is the bandgap of bulk Si and the $d_{i}$ is the diameter of the NW indexed by $i$. Here $C$ and $\alpha$ are fitting parameters inherited from Eq. (1). Within the notion of generalized mean, ${ }^{33}$ the exponent $\beta$ governs the contributions of each $\mathrm{NW}$, namely, the larger the parameter $\beta$, the higher the contribution of the thickest NW to the bandgap. Ultimately the specific value of $\beta$ is an outcome of the material-dependent atomic potentials and hence the quantum size effect. For a single NW $(N=1)$, Eq. (2) reduces to Eq. (1) which suggests that $\{C, \alpha\}$ of single NWs as in Table I can also be used for Eq. (2). Regarding the sensitivity to $\beta$, the estimated bandgap varies only by $\pm 0.1 \mathrm{eV}$ when $\beta$ changes in the range 4-7. Based on our directional analysis, we suggest to use $\beta=5.5$ for $\langle 110\rangle,\langle 111\rangle$, and $\langle 112\rangle$ crossings whereas for $\langle 100\rangle$ crossings $\beta=4$ yields a better estimation. Figure 4 shows the

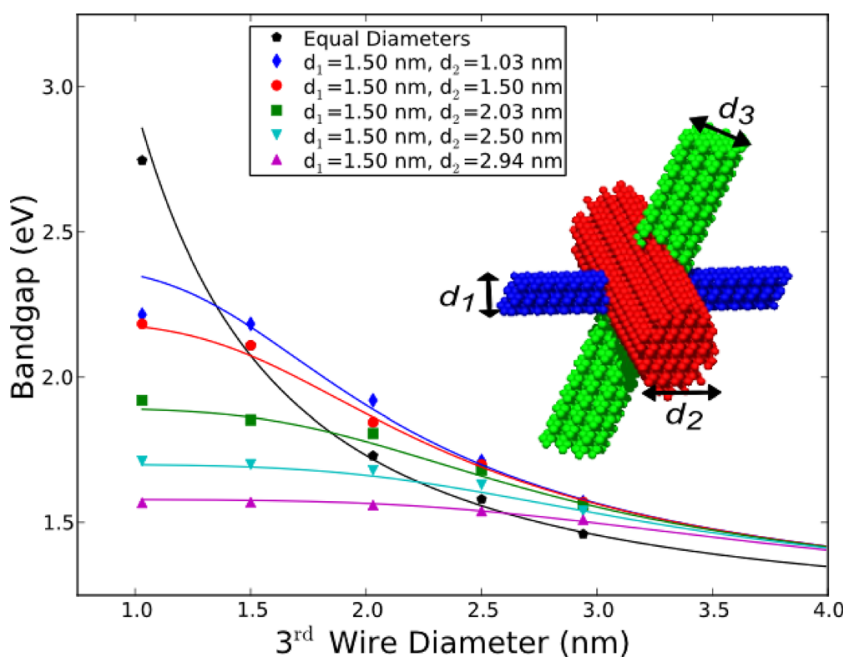

FIG. 4. Bandgap values for three-wire crossings. The calculated data points are shown with the markers; the lines are obtained via Eq. (2) ( $\beta=5.5$ is used, see also Table I). The crossing wire alignments are along $\langle 110\rangle$ directions.

calculated data points and corresponding plots of Eq. (2) for three-dimensional networks $(N=3)$, for $\beta=5.5$. The overall performance of Eq. (2) is highly satisfactory with the anticipated deviations for the very small diameters as we discussed in the single NW case.

In order to estimate $\mathrm{VB}$ and $\mathrm{CB}$ offsets of networks with respect to bulk $\mathrm{Si}$, the form of Eq. (2) can again be invoked as done for the single NW case. Corresponding plots are given in the supplementary material ${ }^{33}$ employing $\left\{C_{\mathrm{VBE}}, \alpha_{\mathrm{VBE}}\right\}$ and $\left\{C_{\mathrm{CBE}}, \alpha_{\mathrm{CBE}}\right\}$ parameters of single Si NWs (given in Table I) together with the same $\beta$ values of bandgap estimation.

In conclusion, we computed the electronic bandgap energies of Si NW structures embedded into silica using an atomistic pseudopotential approach. First, we investigated the variation of bandgap and band edge alignments as a function of wire diameter for various orientations of Si NWs. Our results indicate a bandgap anisotropy that differs from the $\mathrm{H}$ passivated case. After establishing the single-wire case, we extended our consideration to the main subject of this paper, the two- and three-dimensional SiNets. Based on a comprehensive analysis, we proposed an expression to estimate the bandgap values of networks as a function of crossing wire diameters. The form of the expression should, in principle, hold for other materials as well, to assist bandgap engineering of NW networks. This expression can also be used to calculate the valence and conduction band edge alignments with respect to bulk $\mathrm{Si}$. The semiempirical atomistic calculations given in this work are for relatively large diameters. A complimentary follow-up could be a first-principles investigation for small-diameter networks to shed light especially on surface chemistry and strain effects.

We would like to thank Oğuz Gülseren for valuable discussions. This work was supported by The Scientific and Technological Research Council of Turkey (TÜBITAK) with Project No. 109R037 and German Federal Ministry of Education and Research (BMBF) with Project No. TUR09240. Ü.K. acknowledges Helmholtz-Zentrum Dresden - Rossendorf for supporting his visits in Dresden. 
${ }^{1}$ W. Lu, P. Xie, and C. M. Lieber, IEEE Trans. Electron Devices 55, 2859 (2008).

${ }^{2}$ A. M. Morales and C. M. Lieber, Science 279, 208 (1998).

${ }^{3}$ J. D. Holmes, K. P. Johnston, R. C. Doty, and B. A. Korgel, Science 287, 1471 (2000).

${ }^{4}$ Y. Cui, L. J. Lauhon, M. S. Gudiksen, J. Wang, and C. M. Lieber, Appl. Phys. Lett. 78, 2214 (2001).

${ }^{5}$ D. D. D. Ma, C. S. Lee, F. C. K. Au, S. Y. Tong, and S. T. Lee, Science 299, 1874 (2003).

${ }^{6}$ Y. Wu, Y. Cui, L. Huynh, C. J. Barrelet, D. C. Bell, and C. M. Lieber, Nano Lett. 4, 433 (2004).

${ }^{7}$ L. Manna, D. Milliron, A. Meisel, E. Scher, and A. Alivisatos, Nature Mater. 2, 382 (2003).

${ }^{8}$ D. Wang, F. Qian, C. Yang, Z. Zhong, and C. M. Lieber, Nano Lett. 4, 871 (2004).

${ }^{9}$ K. Jun and J. M. Jacobson, Nano Lett. 10, 2777 (2010).

${ }^{10}$ X. Jiang, B. Tian, J. Xiang, F. Qian, G. Zheng, H. Wang, L. Mai, and C. Lieber, Proc. Natl. Acad. Sci. U.S.A. 108, 12212 (2011).

${ }^{11}$ K. Dick, K. Deppert, M. Larsson, T. Mårtensson, W. Seifert, L. Wallenberg, and L. Samuelson, Nature Mater. 3, 380 (2004).

${ }^{12}$ J. Zhou, Y. Ding, S. Deng, L. Gong, N. Xu, and Z. Wang, Adv. Mater. 17, 2107 (2005).

${ }^{13}$ K. A. Dick, K. Deppert, L. S. Karlsson, W. Seifert, L. R. Wallenberg, and L. Samuelson, Nano Lett. 6, 2842 (2006).

${ }^{14}$ M. Totaro, P. Bruschi, and G. Pennelli, Microelectron. Eng. 97, 157 (2012).

${ }^{15}$ P. Serre, C. Ternon, V. Stambouli, P. Periwal, and T. Baron, Sens. Actuators B 182, 390 (2013).

${ }^{16}$ E. Mulazimoglu, S. Coskun, M. Gunoven, B. Butun, E. Ozbay, R. Turan, and H. E. Unalan, Appl. Phys. Lett. 103, 083114 (2013).

${ }^{17}$ X. Zhao, C. M. Wei, L. Yang, and M. Y. Chou, Phys. Rev. Lett. 92, 236805 (2004).

${ }^{18}$ M. Bruno, M. Palummo, A. Marini, R. Del Sole, and S. Ossicini, Phys. Rev. Lett. 98, 036807 (2007).

${ }^{19}$ J.-A. Yan, L. Yang, and M. Y. Chou, Phys. Rev. B 76, 115319 (2007).

${ }^{20}$ L. Huang, N. Lu, J.-A. Yan, M. Y. Chou, C.-Z. Wang, and K.-M. Ho, J. Phys. Chem. C 112, 15680 (2008).
${ }^{21}$ R. Rurali, B. Aradi, T. Frauenheim, and A. Gali, Phys. Rev. B 76, 113303 (2007).

${ }^{22}$ M.-F. Ng, L. Zhou, S.-W. Yang, L. Y. Sim, V. B. C. Tan, and P. Wu, Phys. Rev. B 76, 155435 (2007).

${ }^{23}$ H. Scheel, S. Reich, and C. Thomsen, Phys. Status Solidi B 242, 2474 (2005).

${ }^{24}$ Y. M. Niquet, A. Lherbier, N. H. Quang, M. V. Fernández-Serra, X. Blase, and C. Delerue, Phys. Rev. B 73, 165319 (2006).

${ }^{25}$ J. Kim and M. V. Fischetti, J. Appl. Phys. 110, 033716 (2011).

${ }^{26}$ R. Rurali, Rev. Mod. Phys. 82, 427 (2010).

${ }^{27}$ M. Menon, E. Richter, I. Lee, and P. Raghavan, J. Comput. Theor. Nanosci. 4, 252 (2007).

${ }^{28}$ P. V. Avramov, L. A. Chernozatonskii, P. B. Sorokin, and M. S. Gordon, Nano Lett. 7, 2063 (2007).

${ }^{29}$ G. Bester, J. Phys.: Condens. Matter 21, 023202 (2009).

${ }^{30}$ L.-W. Wang, A. Franceschetti, and A. Zunger, Phys. Rev. Lett. 78, 2819 (1997).

${ }^{31}$ L.-W. Wang and A. Zunger, Phys. Rev. B 59, 15806 (1999).

${ }^{32}$ C. Bulutay, Phys. Rev. B 76, 205321 (2007).

${ }^{33}$ See supplementary material at http://dx.doi.org/10.1063/1.4830039 for the details of the implementation of LCBB method, pseudopotential form factors, generalized mean, and estimation expression for valence and conduction band offsets.

${ }^{34}$ H. Yildirim and C. Bulutay, Phys. Rev. B 78, 115307 (2008).

${ }^{35}$ K. Imakita, M. Ito, M. Fujii, and S. Hayashi, J. Appl. Phys. 105, 093531 (2009).

${ }^{36}$ C. Bulutay, M. Kulakci, and R. Turan, Phys. Rev. B 81, 125333 (2010).

${ }^{37}$ C. Delerue, G. Allan, and M. Lannoo, Phys. Rev. B 48, 11024 (1993).

${ }^{38}$ L. E. Brus, J. Chem. Phys. 79, 5566 (1983).

${ }^{39}$ T. Takagahara and K. Takeda, Phys. Rev. B 46, 15578 (1992).

${ }^{40}$ This dual character of the valence band edge exponents is preserved if we try the form $C_{1} d^{-1}+C_{2} d^{-2}$ as suggested in Ref. 43 .

${ }^{41}$ R. J. Bondi, S. Lee, and G. S. Hwang, ACS Nano 5, 1713 (2011).

${ }^{42}$ V. Schmidt, S. Senz, and U. Gösele, Nano Lett. 5, 931 (2005).

${ }^{43}$ S. Ossicini, C. Bertoni, M. Biagini, A. Lugli, G. Roma, and O. Bisi, Thin Solid Films 297, 154 (1997). 